\title{
Crescimento de Pistia stratiotes em diferentes condições de temperatura e fotoperíodo ${ }^{1}$
}

\author{
Leonardo Farage Cancian², Antonio Fernando Monteiro Camargo ${ }^{3,5}$ e Gustavo Henrique Gonzaga Silva ${ }^{4}$
}

\author{
Recebido em 26/11/2007. Aceito em 23/10/2008
}

\begin{abstract}
RESUMO - (Crescimento de Pistia stratiotes em diferentes condições de temperatura e fotoperíodo). A temperatura e fotoperíodo são variáveis abióticas que influenciam diretamente a produção primária das macrófitas aquáticas, determinam a distribuição geográfica das espécies e afetam a estrutura das comunidades. O objetivo desse trabalho foi avaliar a influência de diferentes condições de temperatura e fotoperíodo no crescimento de Pistia stratiotes. Indivíduos de $P$. stratiotes foram acondicionados em câmaras climáticas, com controle de temperatura e fotoperíodo. Utilizou-se oito tratamentos, $1\left(15^{\circ} \mathrm{C} / 8 \mathrm{~h}\right.$ de fotoperíodo $) ; 2\left(15^{\circ} \mathrm{C} / 12 \mathrm{~h}\right) ; 3\left(25^{\circ} \mathrm{C} / 8 \mathrm{~h}\right) ; 4\left(25^{\circ} \mathrm{C} / 12 \mathrm{~h}\right) ; 5$ $\left(30^{\circ} \mathrm{C} / 8 \mathrm{~h}\right)$ e $6\left(30^{\circ} \mathrm{C} / 12 \mathrm{~h}\right)$. O maior ganho de biomassa ocorreu no tratamento 4 , que apresentou diferenças significativas $(\mathrm{p}<0,05)$ em relação aos tratamentos 1,5 e 6 . Nos tratamentos 1,5 e 6 , a biomassa final foi inferior à biomassa inicial de $P$. stratiotes. A maior redução de biomassa ocorreu no tratamento 6 , que foi significativamente inferior aos tratamentos 2, 3 e 4 . Observou-se nos tratamentos com temperatura de $25^{\circ} \mathrm{C}$ a maior produção de brotos, enquanto que, nos tratamentos com temperatura de $15{ }^{\circ} \mathrm{C}$ e $30^{\circ} \mathrm{C}$, essa produção foi muito reduzida. Concluiu-se que temperatura e fotoperíodo influenciam o crescimento de Pistia stratiotes e que esta espécie apresentou maior crescimento quando submetida à temperatura de $25^{\circ} \mathrm{C}$ e fotoperíodo de 12 horas.
\end{abstract}

Palavras-chave: efeito de fotoperíodo, efeito de temperatura, macrófita aquática

\begin{abstract}
Growth of Pistia stratiotes in different temperature and photoperiod regimes). Temperature and photoperiod are abiotic variables that directly influence aquatic macrophyte production. They also determine the geographic distribution of the species and affect community structure. The aim of this study was to evaluate the growth of Pistia stratiotes in different temperature and photoperiod regimes. $P$. stratiotes plants were conditioned in growth chambers with controlled temperature and photoperiod. Six treatments were used: $1\left(15^{\circ} \mathrm{C} / 8 \mathrm{~h}\right.$ photoperiod $) ; 2\left(15^{\circ} \mathrm{C} / 12 \mathrm{~h}\right) ; 3\left(25^{\circ} \mathrm{C} / 8 \mathrm{~h}\right) ; 4\left(25^{\circ} \mathrm{C} / 12 \mathrm{~h}\right) ; 5\left(30^{\circ} \mathrm{C} / 8 \mathrm{~h}\right)$ and $6\left(30^{\circ} \mathrm{C} / 12 \mathrm{~h}\right)$. The highest biomass gain occurred with treatment number 4 which showed significant differences $(\mathrm{p}<0.05)$ compared to treatments number 1,5 and 6 . In treatments 1, 5 and 6 the final biomass was lower than the initial biomass of $P$. stratiotes. The greatest biomass reduction occurred in treatment number 6 which was significantly lower than in treatments 2,3 and 4 . The highest sprout production was observed in treatments at $25{ }^{\circ} \mathrm{C}$, while in treatments at $15{ }^{\circ} \mathrm{C}$ and $30{ }^{\circ} \mathrm{C}$ sprouting was greatly reduced. We conclude that temperature and photoperiod influence $P$. stratiotes growth and higher growth rates occurred at $25{ }^{\circ} \mathrm{C}$ and 12-hour photoperiod.
\end{abstract}

Key words: aquatic macrophytes, photoperiod effect, temperature effect

\section{Introdução}

Uma das questões mais freqüentes no estudo da ecologia de macrófitas aquáticas é estabelecer os fatores que determinam o seu crescimento, para que se possa prever a ocorrência e abundância das espécies. Os modelos básicos de distribuição e crescimento da vegetação aquática geralmente são explicados pelas relações fisiológicas entre as plantas e as condições ambientais, além da tolerância e habilidade desses vegetais em crescer nas diferentes condições. O crescimento de macrófitas aquáticas está relacionado principalmente com luminosidade, temperatura, disponibilidade de nutrientes, $\mathrm{pH}$, alcalinidade, salinidade, variação no nível de água e na velocidade da corrente, e interações ecológicas, tais como competição e herbivoria (Barendregt \& Bio 2003; Neiff \& Neiff 2003; Henry-Silva \& Camargo 2005). Estas variáveis abióticas e bióticas atuam em conjunto sobre a população ou sobre um indivíduo.

Espécies de macrófitas aquáticas flutuantes, em geral, possuem grande plasticidade fenotípica, reprodução vegetativa intensa e elevada taxa de crescimento (Rubim \& Camargo 2001; Henry-Silva et al. 2008). Estas características, quando associadas a

\footnotetext{
1 Parte da Dissertação de Mestrado do primeiro Autor

2 Universidade Estadual Paulista, Centro de Aqüicultura, Via de Acesso Prof. Paulo Donato Castellane s.n., 14884-900 Jaboticabal, SP, Brasil

3 Universidade Estadual Paulista, Departamento de Ecologia, Avenida 24A, 1515, 13506-900 Rio Claro, SP, Brasil

4 Universidade Federal Rural do Semi-Árido, Departamento de Ciências Animais, BR 110 - Km 47, Presidente Costa e Silva, 59625-900 Mossoró, RN, Brasil

5 Autor para correspondência: afmc@rc.unesp.br
} 
condições favoráveis, freqüentemente resultam em proliferação indesejada destes vegetais. A proliferação indesejada pode acarretar prejuízo aos diversos usos dos recursos hídricos, tais como a obstrução de canais de irrigação, prejuízo à navegação e atividades de recreação, redução da capacidade de geração de energia elétrica e comprometimento da atividade pesqueira (Gopal 1990; Seshavatharam 1990; Vereecken et al. 2006).

A temperatura e o fotoperíodo são variáveis abióticas que influenciam diretamente na produção primária das macrófitas aquáticas (Santamaria \& Van Vierssen 1997), determinam a distribuição geográfica das espécies e afetam a estrutura das comunidades (Svensson \& WigrenSvensson 1992; Rooney \& Kalff 2000). O aquecimento global causado por ações antrópicas tem modificado sistemas biológicos. As mudanças incluem alterações em eventos fenológicos (por exemplo, brotamento de folhas, data de floração, migração e tempo de reprodução), distribuição de espécies e estrutura da comunidade (Rosenzweig et al. 2008). Esse aquecimento, portanto, também pode afetar o crescimento de macrófitas aquáticas.

Pistia stratiotes (Araceae) é uma macrófita aquática flutuante livre, com distribuição cosmopolita tropical (Pott \& Pott 2000). É considerada uma espécie que prolifera indesejadamente em alguns ecossistemas aquáticos tropicais. Pode-se destacar como exemplos de crescimento indesejado de $P$. stratiotes a proliferação em canais de irrigação em Gâmbia (Terry 1981), em um braço do reservatório de Itaipu, Brasil (Thomaz et al. 1999) e em viveiros de criação de peixes na África (Petr 2000). No Brasil, os maiores prejuízos decorrentes de sua proliferação são causados nas áreas mais quentes e em mananciais poluídos (Lorenzi 1982). Alterações antropogênicas, tais como a eutrofização e o aquecimento global, podem promover o crescimento de $P$. stratiotes e provocar a expansão de sua área de distribuição.

Nesse contexto, este trabalho tem como objetivo principal avaliar a influência da temperatura e do fotoperíodo no crescimento da macrófita aquática flutuante Pistia stratiotes.

\section{Material e métodos}

O experimento foi realizado no Laboratório de Controle Biológico de Plantas Daninhas, localizado na Universidade Estadual Paulista Júlio de Mesquita Filho, campus de Jaboticabal ( $21^{\circ} 15^{\prime} 22^{\prime \prime}$ S e $\left.48^{\circ} 18^{\prime} 58^{\prime \prime} \mathrm{W}\right)$. Indivíduos de Pistia stratiotes foram coletados no rio Aguapeú, que pertence à bacia hidrográfica do rio Itanhaém, litoral sul do Estado de São Paulo

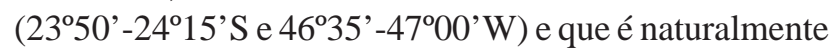
colonizado por várias espécies de macrófitas aquáticas, incluindo P. stratiotes. Dois indivíduos de tamanho e aparência semelhantes foram acondicionados em cada unidade experimental retangular $(27 \times 18 \mathrm{~cm})$ de $4 \mathrm{~L}$ de capacidade, contendo solução nutritiva de Hoagland \& Arnon (1950) diluída a $10 \%$ e com a seguinte concentração de nutrientes: $\mathrm{NH}_{4}-\mathrm{N}=10,5 \mathrm{mg} \mathrm{L}^{-1}$; $\mathrm{NO}_{3}-\mathrm{N}=10,5 \mathrm{mg} \mathrm{L}^{-1} ; \mathrm{PO}_{4}-\mathrm{P}=3,1 \mathrm{mg} \mathrm{L}^{-1} ; \mathrm{K}=23 \mathrm{mg} \mathrm{L}^{-1}$; $\mathrm{Ca}=20 \mathrm{mg} \mathrm{L}^{-1} ; \mathrm{Mg}=5,9 \mathrm{mg} \mathrm{L}^{-1} ; \mathrm{Fe}=0,6 \mathrm{mg} \mathrm{L}^{-1}$; $\mathrm{H}_{3} \mathrm{BO}_{3}=2,68 \mathrm{mg} \mathrm{L}^{-1} ; \mathrm{MnCl}_{2} 4 \mathrm{H}_{2} \mathrm{O}=1,81 \mathrm{~g} \mathrm{~L}^{-1} ; \mathrm{ZnSO}_{4}$ $7 \mathrm{H}_{2} \mathrm{O}=0,22 \mathrm{~g} \mathrm{~L}^{-1} ; \mathrm{CuSO}_{4} 5 \mathrm{H}_{2} \mathrm{O}=0,08 \mathrm{~g} \mathrm{~L}^{-1} ; \mathrm{H}_{2} \mathrm{Mo}_{2}$ $4 \mathrm{H}_{2} \mathrm{O}=0,02 \mathrm{~g} \mathrm{~L}^{-1}$.

Utilizou-se um delineamento experimental bifatorial (temperatura e fotoperíodo), com 3 e 2 níveis, respectivamente. $\mathrm{O}$ experimento consistiu de seis tratamentos e três réplicas. As unidades experimentais foram acondicionadas em câmaras climáticas, com controle de temperatura e fotoperíodo. A radiação no interior das incubadoras foi determinada com um radiômetro da marca Li-cor e apresentou valores médios e desvios padrão de $32,04 \mu \mathrm{mol} . \mathrm{s}^{-1} \cdot \mathrm{m}^{-2}$ e $4,89 \mu \mathrm{mol} . \mathrm{s}^{-1} \cdot \mathrm{m}^{-2}$, respectivamente. Os tratamentos utilizados foram: 1 $\left(15^{\circ} \mathrm{C}\right.$ e 8 horas de fotoperíodo); $2\left(15^{\circ} \mathrm{C}\right.$ e 12 horas de fotoperíodo); $3\left(25^{\circ} \mathrm{C}\right.$ e 8 horas de fotoperíodo); $4\left(25^{\circ} \mathrm{C}\right.$ e 12 horas de fotoperíodo); 5 ( $30^{\circ} \mathrm{C}$ e 8 horas de fotoperíodo) e $6\left(30{ }^{\circ} \mathrm{C}\right.$ e 12 horas de fotoperíodo).

$\mathrm{O}$ experimento teve duração de trinta dias e, a intervalos de três dias, a massa fresca de cada unidade era obtida da seguinte forma: os indivíduos eram retirados das unidades experimentais e colocados sobre uma peneira durante cinco minutos para eliminar o excesso de água (conforme Agami \& Reddy 1990). Em seguida, a massa fresca era obtida por meio de pesagem em uma balança marca Bel (modelo Mark 330, com precisão de centésimo de grama), posteriormente, os indivíduos eram recolocados nas unidades experimentais. A massa fresca inicial média de $P$. stratiotes colocada em cada unidade amostral foi de $6,37 \pm 1,73 \mathrm{~g}$. O número de indivíduos de $P$. stratiotes nas unidades experimentais também foi obtido em cada período de três dias. Considerou-se indivíduo a roseta, independente de estar ligada por um rizoma à planta mãe ou não. Neste mesmo intervalo de tempo, a solução nutritiva era totalmente substituída para que as concentrações de nutrientes das unidades experimentais fossem mantidas elevadas. A massa seca de P. stratiotes foi calculada a partir da equação de regressão linear entre a massa fresca (MF) e a massa seca (MS) de indivíduos da mesma população coletada no rio Aguapeú, mas não utilizados no experimento.

$$
\mathrm{MS}=\mathrm{MF} / 5,986 \quad(\mathrm{r}=0,9917 ; \mathrm{n}=50)
$$

Ao final do experimento, foi calculada a diferença entre as biomassas final e inicial para todas as unidades experimentais e, em seguida, aplicou-se a análise de variância (ANOVA) bifatorial para os fatores temperatura 
e fotoperíodo, com 3 e 2 níveis respectivamente, e teste a posteriori de Tukey (Zar 1999), para verificar a ocorrência de diferenças significativas entre os tratamentos. O nível de significância considerado para todos os testes foi 0,05 .

\section{Resultados}

O ganho de massa em $P$. stratiotes nos diferentes tratamentos foi bastante distinto. Nos tratamentos 2, 3 e 4, observou-se ganho de massa nos 30 dias de experimento, enquanto que nos tratamentos 1,5 e 6 , observou-se redução da fitomassa (Fig. 1). A ANOVA fatorial mostrou a ocorrência de diferenças significativas entre o ganho de massa nas diferentes temperaturas $(\mathrm{GL}=2 ; F=22,142 ; \mathrm{p}=0,00009)$ e na interação entre temperatura e fotoperíodo $(\mathrm{GL}=2 ; F=6,397 ; \mathrm{p}=0,012)$.

Nos tratamentos 1,5 e 6 , a biomassa final foi inferior à biomassa inicial de $P$. stratiotes. $\mathrm{O}$ maior ganho de biomassa ocorreu no tratamento $4\left(25^{\circ} \mathrm{C}\right.$ e 12 horas), que foi significativamente superior aos tratamentos 1 $(\mathrm{p}=0,009), 5(\mathrm{p}=0,023)$ e $6(\mathrm{p}=0,0008)$, nos quais se observou redução de biomassa (Tab. 1). A maior redução de biomassa ocorreu no tratamento $6\left(30^{\circ} \mathrm{C}\right.$ e 12 horas) que foi significativamente $(\mathrm{p}<0,05)$ inferior aos tratamentos $2(\mathrm{p}=0,007), 3(\mathrm{p}=0,001)$ e 4 $(\mathrm{p}=0,0008)$, mas que não apresentou diferenças significativas em relação ao tratamento $1(\mathrm{p}=0,59)$ e 5 $(\mathrm{p}=0,32)($ Tab. 1).

A quantidade de indivíduos também foi bastante distinta entre os tratamentos (Fig. 2). A ANOVA fatorial aplicada ao número de indivíduos mostrou a ocorrência de diferenças significativas entre o número final de indivíduos nas diferentes temperaturas $(\mathrm{GL}=2 ; F=68,379$; $\mathrm{p}=0,00000$ ), fotoperíodo ( $\mathrm{GL}=1 ; F=16,0049 ; \mathrm{p}=0,0017$ ) e para a interação entre temperatura e fotoperíodo $(\mathrm{GL}=2$; $F=28,921 ; \mathrm{p}=0,00002)$. Nos tratamentos com temperatura de $15^{\circ} \mathrm{C}$, a quantidade de indivíduos foi pequena, destacando-se a manutenção da quantidade inicial de indivíduos no fotoperíodo de 12 horas. Nos tratamentos com temperatura de $25{ }^{\circ} \mathrm{C}$, observou-se as maiores quantidades de indivíduos no final do experimento. No tratamento $4\left(25^{\circ} \mathrm{C}\right.$ e 12 horas $)$ o número de indivíduos no final do experimento foi de 761 indivíduos. $\mathrm{m}^{-2}$. O número de indivíduos nos tratamentos 5 e $6\left(30^{\circ} \mathrm{C}\right)$ foi semelhante ao tratamento $3\left(25^{\circ} \mathrm{C} ; 8\right.$ horas) (Tab. 2). Destaca-se que o tamanho médio dos indivíduos em todos os tratamentos foi bastante reduzido.

\section{Discussão}

Constatamos que $P$. stratiotes é sensível a baixas temperaturas, pois apresentou menor crescimento, bem como amarelecimento e morte de folhas mais externas da roseta quando submetida à temperatura de $15^{\circ} \mathrm{C}$. Mazzeo et al. (1993) também observaram no reservatório Del Cisne no Uruguai, a morte de folhas e a diminuição no tamanho dos indivíduos de $P$. stratiotes entre os meses de junho e setembro, devido às baixas temperaturas da água superficial (mínima de $9{ }^{\circ} \mathrm{C}$ ) e à ocorrência de geadas. Šajna et al. (2007), em um estudo sobre a expansão e a sobrevivência de $P$. stratiotes em um riacho termal da Eslovênia, demonstraram que as
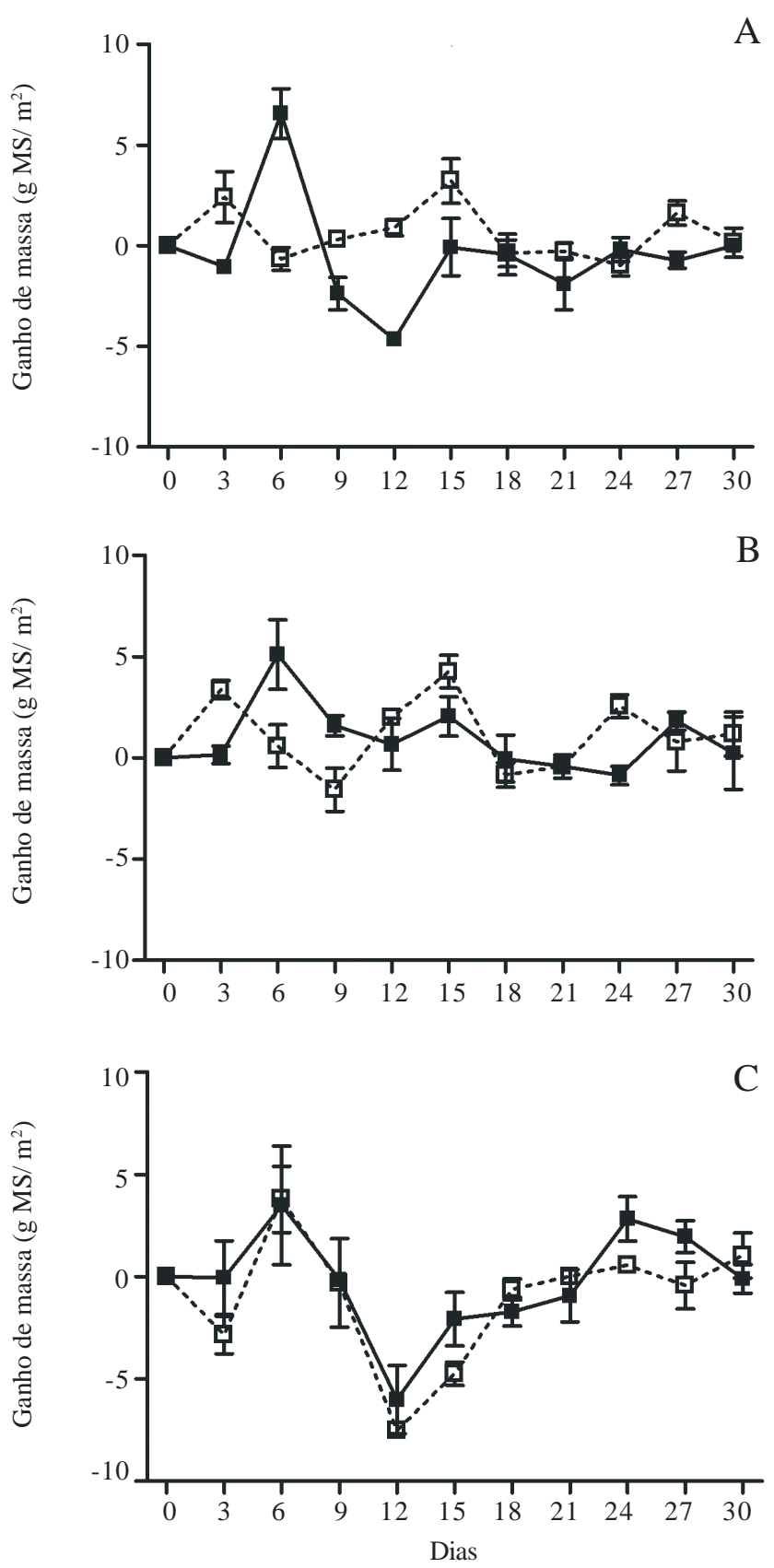

Figura 1. Média e desvio padrão de ganhos de biomassa $\left(\mathrm{g} \mathrm{MS} / \mathrm{m}^{2}\right) \mathrm{em}$ Pistia stratiotes nos tratamentos $1\left(15^{\circ} \mathrm{C} ; 8\right.$ horas $)$ e $2\left(15^{\circ} \mathrm{C} ; 12\right.$ horas): A; $3\left(25^{\circ} \mathrm{C} ; 8\right.$ horas $)$ e $4\left(25^{\circ} \mathrm{C} ; 12\right.$ horas $): \mathrm{B} ; 5\left(30{ }^{\circ} \mathrm{C} ; 8\right.$ horas) e $6\left(30^{\circ} \mathrm{C}\right.$; 12 horas $)$ - C. ( $-\square-=8$ horas; -- $\square--=12$ horas $)$. 
Tabela 1. Valores médios da diferença entre biomassa final e inicial nos seis tratamentos (indicados entre colchetes) e valores de $\mathrm{p}$ do teste de Tukey aplicado aos valores de diferença de biomassa entre tratamentos $(\mathrm{QMr}=22,133 ; \mathrm{GLr}=12)$. O asterisco indica diferenças significativas $(\mathrm{p} \leq 0,05)$.

\begin{tabular}{|c|c|c|c|c|c|c|c|c|}
\hline & Temperatura & Fotoperíodo & $\begin{array}{c}{[1]} \\
-4,791\end{array}$ & $\begin{array}{c}{[2]} \\
6,3293\end{array}$ & $\begin{array}{c}{[3]} \\
10,327\end{array}$ & $\begin{array}{c}{[4]} \\
11,901\end{array}$ & $\begin{array}{c}{[5]} \\
-2,739\end{array}$ & $\begin{array}{c}[6\}] \\
-11,05\end{array}$ \\
\hline 1 & 15 & 8 & - & & & & & \\
\hline 2 & 15 & 12 & 0,107428 & - & & & & \\
\hline 3 & 25 & 8 & $0,018970 *$ & 0,895032 & - & & & \\
\hline 4 & 25 & 12 & $0,009572 *$ & 0,698782 & 0,998151 & - & & \\
\hline 5 & 30 & 8 & 0,993552 & 0,243483 & $0,046640 *$ & $0,023389 *$ & - & \\
\hline 6 & 30 & 12 & 0,596553 & $0,007124^{*}$ & $0,001431 *$ & $0,000830 *$ & 0,320285 & - \\
\hline
\end{tabular}

temperaturas da água no mês de agosto $\left(24\right.$ a $\left.28{ }^{\circ} \mathrm{C}\right)$ foram favoráveis ao crescimento, enquanto que as temperaturas do ar no inverno $\left(16^{\circ} \mathrm{C}\right.$ na altura da roseta) foram desfavoráveis.

Os estudos sobre o efeito de altas temperaturas no crescimento de macrófitas aquáticas flutuantes são raros e nossos resultados indicam que altas temperaturas também são desfavoráveis para $P$. stratiotes $\left(30^{\circ} \mathrm{C}\right)$ pois, embora o número de indivíduos tenha aumentado, a biomassa diminuiu em decorrência do tamanho reduzido dos brotos novos. O efeito negativo de temperaturas elevadas sobre macrófitas aquáticas flutuantes também foi observado por van der Heide et al. (2006a), que verificaram maior crescimento de S. molesta e Lemna minor em temperaturas entre 25 e $30^{\circ} \mathrm{C}$, e crescimento reduzido em temperaturas de 15 e $35^{\circ} \mathrm{C}$. Destacamos, no entanto, que $P$. stratiotes é mais sensível a altas temperaturas do que $L$. minor, pois esta última somente teve seu crescimento reduzido em temperaturas de $35^{\circ} \mathrm{C}$ (van der Heide et al. 2006a).

É importante destacar que P. stratiotes é uma espécie provavelmente originária da América do Sul e que atualmente se distribui por todas as regiões tropicais e subtropicais do Planeta (Howard \& Harley 1998). No entanto, esta macrófita aquática também pode ocorrer em regiões temperadas, em ambientes submetidos à poluição térmica ou em fontes termais (Šajna et al. 2007). Desta forma, com a perspectiva do aquecimento global, que tem influenciado a distribuição de espécies (Rosenzweig et al. 2008), P. stratiotes poderá se deslocar de regiões de menores latitudes para as de maiores, reduzindo sua abundância nos trópicos e aumentando-a nos subtrópicos. No entanto, mudanças climáticas globais não afetam individualmente uma espécie, mas também as espécies que interagem com ela. Os herbívoros que se alimentam de $P$. stratiotes provavelmente terão sua atividade alterada com as mudanças de temperatura. Destacamos que van der Heide et al. (2006b) observaram que a pressão de herbivoria do lepidoptero Cataclysta lemnata sobre Lemna minor aumentou entre temperaturas de 15 a $24^{\circ} \mathrm{C}$. Portanto, a previsão sobre a distribuição e o crescimento de macrófitas aquáticas devido ao aquecimento global torna-se difícil, devido a diversos fatores compensatórios envolvidos.

O fotoperíodo também influenciou o crescimento de $P$. stratiotes, principalmente quando cultivada em temperatura de $15^{\circ} \mathrm{C}$. Nesta temperatura e sob fotoperíodo de 12 horas, o crescimento foi pequeno, enquanto que no fotoperíodo de 8 horas, houve uma redução na biomassa. Provavelmente, a temperatura mais baixa e a menor quantidade de radiação produziram um efeito conjunto, desfavorecendo o crescimento desta macrófita, sendo que nos demais tratamentos, o fotoperíodo não causou efeito sobre o ganho de biomassa. Vale ressaltar que o efeito de fotoperíodo sobre macrófitas aquáticas tem sido pouco estudado, no entanto, Pilon \& Santamaría (2002) observaram que a macrófita aquática submersa Potamogeton pectinatus também apresentou diferenças no ganho de biomassa, relacionado com o fotoperíodo. Estes autores verificaram maior ganho de massa em fotoperíodos longos. No entanto, essa espécie habita regiões temperadas, enquanto que $P$. stratiotes é uma macrófita que habita principalmente regiões tropicais, sendo que o efeito do fotoperíodo pode ser diferente para ambas.

Os resultados aqui obtidos mostraram grandes diferenças no número de indivíduos ao final do experimento, nos diferentes tratamentos. $\mathrm{O}$ maior número de indivíduos ocorreu nas condições de $25^{\circ} \mathrm{C}$ e 12 horas de fotoperíodo, quando observamos o maior ganho de massa. No entanto, outros autores observaram maior quantidade de indivíduos em épocas em que o ganho e/ou biomassa não foram os mais elevados. Mazzeo et al. (1993) averiguaram, no lago Del Cisne, Uruguai, que a produção de indivíduos-filhos foi maior entre o início do outono e final do inverno e a maior biomassa foi observada no verão. Em um rio termal da Eslovênia, Šajna et al. (2007a) observaram maior 
Tabela 2. Valores médios do número de indivíduos nos seis tratamentos (indicados entre colchetes) e valores de p do teste de Tukey aplicado ao número médio de indivíduos comparados entre tratamentos ( $\mathrm{QMr}=4775 ; \mathrm{GLr}=12)$. $\mathrm{O}$ asterisco indica diferenças significativas $(\mathrm{p} \leq 0,05)$.

\begin{tabular}{|c|c|c|c|c|c|c|c|c|}
\hline & Temperatura & Fotoperíodo & $\begin{array}{c}{[1]} \\
68,587\end{array}$ & $\begin{array}{c}{[2]} \\
41,152\end{array}$ & $\begin{array}{c}{[3]} \\
281,21\end{array}$ & $\begin{array}{c}{[4]} \\
761,32\end{array}$ & $\begin{array}{c}{[5]} \\
308,64\end{array}$ & $\begin{array}{c}[6\}] \\
246,91\end{array}$ \\
\hline 1 & 15 & 8 & - & & & & & \\
\hline 2 & 15 & 12 & 0,995831 & - & & & & \\
\hline 3 & 25 & 8 & $0,025135^{*}$ & $0,011120^{*}$ & - & & & \\
\hline 4 & 25 & 12 & $0,000159 *$ & $0,000159^{*}$ & $0,000169^{*}$ & - & & \\
\hline 5 & 30 & 8 & $0,011120^{*}$ & $0,005014 *$ & 0,995831 & $0,000190^{*}$ & - & \\
\hline 6 & 30 & 12 & 0,069627 & $0,030861 *$ & 0,988401 & $0,000163 *$ & 0,874703 & - \\
\hline
\end{tabular}
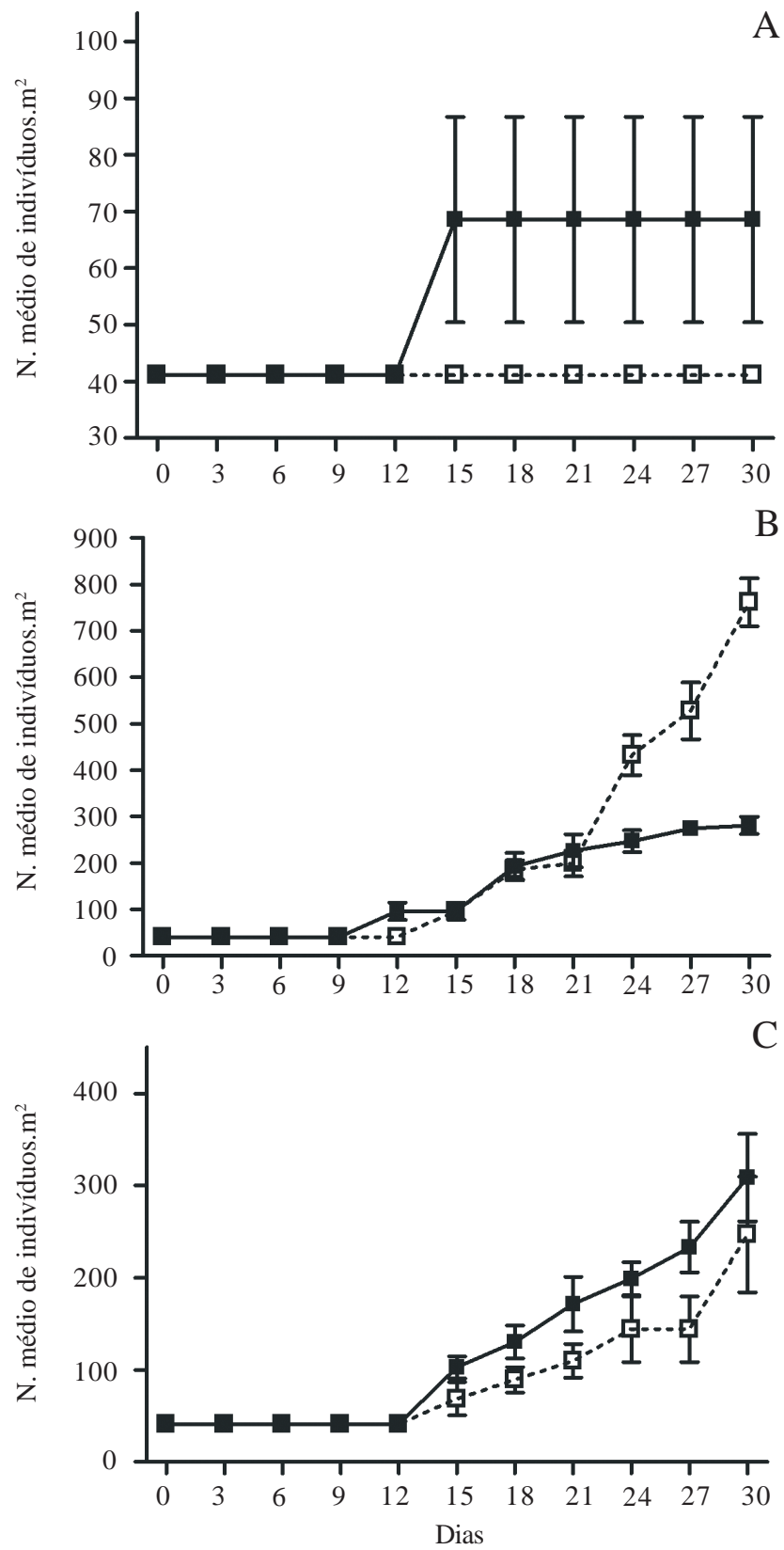

Figura 2. Média e desvio padrão do número de indivíduos. $\mathrm{m}^{-2} \mathrm{em}$ Pistia stratiotes nos tratamentos $1\left(15^{\circ} \mathrm{C} ; 8\right.$ horas $)$ e $2\left(15^{\circ} \mathrm{C} ; 12\right.$ horas): A; $3\left(25^{\circ} \mathrm{C} ; 8\right.$ horas $)$ e $4\left(25^{\circ} \mathrm{C} ; 12\right.$ horas $)$ : $\mathrm{B} ; 5\left(30^{\circ} \mathrm{C} ; 8\right.$ horas) e 6 ( $30^{\circ} \mathrm{C} ; 12$ horas): C. (-ø- = 8 horas; -- $\square--=12$ horas). quantidade de estolões em dezembro (inverno), quando as temperaturas do ar e água estavam menores e a biomassa também era reduzida. Destacamos que esses dois trabalhos foram desenvolvidos em campo, nos quais as condições ambientais, tal como a disponibilidade de nutrientes, também variaram ao longo do ano. É importante salientar que a influência da temperatura, do fotoperíodo e da disponibilidade de nutrientes afeta o ganho de massa e a produção de brotos em $P$. stratiotes, no entanto, ainda se desconhece como isso ocorre.

Podemos concluir que, mesmo para espécie nativa de clima tropical, no qual as temperaturas de 15 e $30^{\circ} \mathrm{C}$ são freqüentes, essas temperaturas podem limitar o crescimento do vegetal. Também concluímos que o aumento de biomassa e a produção de brotos não estão necessariamente relacionados. Neste aspecto, observamos três relações distintas: i) diferenças entre o aumento do número de indivíduos e semelhança no ganho de biomassa, ii) aumento no número de indivíduos e redução de biomassa e iii) aumento de biomassa e manutenção do número de indivíduos.

\section{Agradecimentos}

Os autores agradecem ao Conselho Nacional de Desenvolvimento Científico e Tecnológico (CNPq), pela bolsa de mestrado concedida ao primeiro autor e ao Prof. Dr. Robinson Antonio Pitelli, por gentilmente ceder as instalações do Laboratório de Controle Biológico da UNESP, campus de Jaboticabal, SP.

\section{Referências bibliográficas}

Agami, M. \& Reddy, K. R. 1990. Competition for space between Eichhornia crassipes (Mart.) Solms and Pistia stratiotes L. cultured in nutrient-enriched water. Aquatic Botany 38: 195-208.

Barendregt, A. \& Bio, A.M.F. 2003. Relevant variables to predict macrophytes communities in running waters. Ecological Modelling 160: 205-217.

Gopal, B. 1990. Aquatic weed problems and management in Asia. Pp. 318-340. In: A.H. Pieterse \& K.J. Murphy (eds.). Aquatic Weeds-The Ecology and Management of Nuisance Aquatic Vegetation. Oxford, Oxford University Press. 
Henry-Silva, G.G. \& Camargo, A.F.M. 2005. Interações ecológicas entre as macrófitas flutuantes Eichhornia crassipes e Pistia stratiotes. Hoehnea 32: 445-452.

Henry-Silva, G.G.; Pezzato, M.M. \& Camargo, A.F.M. 2008. Growth of free-floating aquatic macrophytes in different concentrations of nutrients. Hydrobiologia 610: 153-160.

Hoagland, D.R. \& Arnon, D.I. 1950. The water culture method of growing plants without soil. University of California, Berkeley. California Agriculture Experiment Station Circular.

Howard, G.W. \& Harley, K.L.S. 1998. How do floating aquatic weeds affect wetland conservation and development? How can these effects be minimised? Wetland Ecol. Manage. 5: 215-225.

Lorenzi, H. 1982. Plantas daninhas do Brasil: terrestres, aquáticas, parasitas, tóxicas e medicinais. Nova Odessa, Instituto Plantarum.

Mazzeo, N.; Crosa, D. \& Sommaruga, R. 1993. Productividad y variación estacional de la biomasa de Pistia stratiotes $\mathrm{L}$. en el reservorio del Cisne, Uruguay. Acta Limnologica Brasiliensia 6: $186-195$.

Neiff, J.J. \& Poi de Neiff, A.S.G. 2003. Connectivity processes as a basis for the management of aquatic plants. Pp. 39-58. In: Ecologia e Manejo de Macrófitas Aquáticas. S. Thomaz \& L.M. Bini (eds.). Nupélia - Maringá (SC). Eduem, Maringá.

Petr, T. 2000. Interactions between fish and aquatic macrophytes in inland waters. A review. FAO Fishers Technical Paper. 396, Rome. FAO.

Pilon, J. \& Santamaría, L. 2002. Clonal variation in morphological and physiological responses to irradiance and photoperiod for the aquatic angiosperm Potamogeton pectinatus. Journal of Ecology 90: 859-870.

Pott, V.J. \& Pott, A. 2000. Plantas Aquáticas do Pantanal. Corumbá, MS, Embrapa: Centro de Pesquisa Agropecuária do Pantanal.

Rooney, N. \& Kalff, J. 2000. Inter-annual variation in submerged macrophyte community biomass and distribution: the influence of temperature and lake morphometry. Aquatic Botany 68: 321-335
Rosenzweig, C.; Karoly, D.; Vicarelli, M.; Neofotis, P.; Wu, Q.; Casassa, G.; Menzel, A.; Root, T.L.; Estrella, N.; Seguin, B.; Tryjanowski, P.; Liu, C.; Rawlins, S. \& Imeson, A. 2008. Attributing physical and biological impacts to anthropogenic climate change. Nature 453: $353-358$.

Rubim, M.A.L. \& Camargo, A.F.M. 2001. Taxa de crescimento específico da macrófita aquática Salvinia molesta em um braço do rio Preto, Itanhaém, São Paulo. Acta Limnologica Brasiliensia 13: $61-73$.

Šajna, N.; Haler, M.; Škornik, S. \& Kaligariç, M. 2007. Survival and expansion of Pistia stratiotes L. in a thermal stream in Slovenia. Aquatic Botany 87: 75-79

Santamaria \& Van Vierssen, W. 1997. Photosynthetic temperature responses of fresh- and brackish-water macrophytes: a review. Aquatic Botany 58: 135-150.

Seshavatharam, V. 1990. Traditional uses and problem of noxious growth. Pp. 201-218. In: B. Gopal (ed.). Ecology and Management of Aquatic Vegetation in the Indian Subcontinent. Netherlands, Kluwer Academic Publishers.

Svensson, R. \& Wigren-Svensson, M. 1992. Effects of cooling water discharge on the vegetation in the Forsmark Biotest Basin, Sweden. Aquatic Botany 42: 121-141.

Terry, P.J. 1981. Weeds and their control in the Ghambia. Tropical Pest Management 27: 44-52.

Thomaz, S.M.; Bini, L.M.; Souza, M.C.; Kita, K.K. \& Camargo, A.F.M. 1999. Aquatic macrophytes of Itaipu Reservoir, Brazil: survey of species and ecological considerations. Brazilian Archives of Biology and Technology 42: 15-22.

van der Heide, T.; Roijackers, R.M.; Peeters, E.T.H.M. \& van Nes, E.H. 2006b Experiments with duckweed-moth systems suggest that global warming may reduce rather than promote herbivory. Freshwater Biology 51: 110-116.

van der Heide, T.; Roijackers, R.M.; van Nes, E.H. \& Peeters, E.T.H.M. 2006a A simple equation for describing the temperature dependent growth of free-floating macrophytes. Aquatic Botany 84: 171-175.

Vereecken, H.; Baetens, J.; Viaene, P.; Mostaert, F. \& Meire, P. 2006. Ecological management of aquatic plants: effects in lowland streams. Hydrobiologia 570: 205-210.

Zar, J.H. 1999. Biostatistical analysis. $4^{\text {th }}$ ed. New Jersey, Prentice-Hal Inc. 